\title{
SGLT2 inhibitors and diabetic ketoacidosis: data from the FDA Adverse Event Reporting System
}

\author{
Gian Paolo Fadini ${ }^{1} \cdot$ Benedetta Maria Bonora ${ }^{1}$ - Angelo Avogaro ${ }^{1}$
}

Received: 10 March 2017 / Accepted: 11 April 2017/Published online: 12 May 2017

(C) Springer-Verlag Berlin Heidelberg 2017

\begin{abstract}
Aims/hypothesis Sodium-glucose co-transporter-2 inhibitors (SGLT2i) are indicated for the treatment of type 2 diabetes and may also improve glucose control in type 1 diabetes. In 2015, regulatory agencies warned that SGLT2i may favour diabetic ketoacidosis (DKA). We provide a detailed analysis of DKA reports in which an SGLT2i was listed among suspect or concomitant drugs in the US Food and Drug Administration Adverse Event Reporting System (FAERS). Methods We first analysed the entire public FAERS up to September (third quarter [Q3]) 2016 to extract the number of reports, background indications and concomitant medications, and to calculate proportional reporting ratios (PRRs) and safety signals. We then mined single FAERS files from the first quarter (Q1) of 2014 to 2016 Q3 to obtain detailed information on DKA reports.

Results The FAERS database contains $>2500$ DKA reports in which SGLT2i are listed as suspect or concomitant drugs. The PRR of DKA in reports including vs those not including an SGLT2i and having a diabetes indication was $7.9(95 \%$ CI 7.5 , 8.4 ) and was higher for type 1 diabetes. Several concomitant conditions were less prevalent in DKA reports with SGLT2i vs DKA reports filed for other drugs. A detailed analysis of 2397 DKA reports for SGLT2i from 2014 Q1 to 2016 Q3 revealed a predominance of women, an extremely wide range
\end{abstract}

Electronic supplementary material The online version of this article (doi:10.1007/s00125-017-4301-8) contains peer-reviewed but unedited supplementary material, which is available to authorised users.

Gian Paolo Fadini

gianpaolo.fadini@unipd.it

1 Department of Medicine, University of Padova, Via Giustiniani 2, 35128 Padova, Italy of age and body weight, and a highly variable duration of SGLT2i treatment before onset of DKA. In 37 individuals (1.54\%), DKA was fatal.

Conclusions/interpretation Based on the profile of these reports, SGLT2i-associated DKA may not be limited to any particular demographic or comorbid subpopulation and can occur at any duration of SGLT2i use.

Data availability A list of FDA reports analysed in the study is available in the figshare repository, $10.6084 / \mathrm{m} 9$.figshare. 4903211. Other data are available from the corresponding author on reasonable request.

Keywords Case report · Glucose-lowering medications . Pharmacovigilance $\cdot$ Therapy
Abbreviations
DKA Diabetic ketoacidosis
FAERS Food and Drug Administration Adverse Event Reporting System
FDA Food and Drug Administration
PRR Proportional reporting ratio
SGLT2i Sodium-glucose co-transporter-2 inhibitor

\section{Introduction}

By increasing urinary glucose excretion, sodium-glucose cotransporter-2 inhibitors (SGLT2i) lower glycaemia, body weight and blood pressure. Furthermore, the SGLT2i empagliflozin has proven superior to placebo in preventing cardiovascular events and death [1]. Thus, SGLT2i are particularly appealing for the treatment of type 2 diabetes and, due to their insulin-independent mode of action, also for the treatment of type 1 diabetes [2]. In view of the predictably wide 
clinical uptake of SGLT2i, monitoring their adverse events is critical. In addition to the risk of genitourinary tract infections, a safety signal has emerged that SGLT2i can favour diabetic ketoacidosis (DKA) [3, 4], which is otherwise rare in type 2 diabetes. The mechanisms are only partially understood [5] and common triggers may be absent. The risk of DKA is perceived to be higher when SGLT2 $\mathrm{i}$ are used off-label in type 1 diabetes; some reports indicate that SGLT2i-associated DKA occurred in individuals with presumed type 2 diabetes who were subsequently diagnosed with autoimmune diabetes [6]. The number of reports is steadily increasing, but cases described in the literature are unlikely to represent the entire spectrum of this adverse event. We therefore aimed to analyse rates, disproportionality and characteristics of DKA reports in which an SGLT2i was listed among suspect or concomitant drugs in the US Food and Drug Administration (FDA) Adverse Event Reporting System (FAERS).

\section{Methods}

In pharmacovigilance assessment, disproportionality emerges when a specific adverse event is associated with a given drug, whereas in the absence of disproportionality the distributions of reported adverse events are similar across drugs [7]. The FAERS database contains adverse events filed to the FDA from the first quarter (Q1) of 2004 to the third quarter (Q3) of 2016, in a total of 8,554,531 reports. Each report contains demographic characteristics, type of reaction(s) and outcome(s), suspect and concomitant $\operatorname{drug}(\mathrm{s})$, duration of treatment and indication(s) for use. FAERS files are made publicly available on a quarterly basis and are accessible using methods for orthogonal database searches. AERSMine, an open-access web-based data mining tool, is the most comprehensive software to analyse the FAERS up to 2016 Q3 [8].

We searched all DKA reports in which an SGLT2i was listed among suspect or concomitant drugs (hereafter termed 'DKA reports with SGLT2i'), because any DKA occurring in individuals taking SGLT2i should contribute to pharmacovigilance assessment.

We first ran a query on AERSMine spanning the entire FAERS database (see electronic supplementary material [ESM] Methods) to obtain the number of cases, indications and concomitant medications, and to calculate the rates and measures of disproportionality (proportional reporting ratios [PRRs] and safety signals [information components] [7]). After gathering this overall picture, we mined the FAERS database from 2014 Q1 to 2016 Q3 using a custom procedure developed to collect details of each single case. The retrospective search started in 2014 Q1 because the number of reported incidents of SGLT2i-associated DKA was very low before 2014 Q1. We retrieved the following detailed information: date, case identifier, demographic characteristics, country of origin, type of SGLT2i, indication for use, duration of treatment, type of reaction, and outcome (fatal vs non-fatal). The BenjaminiHochberg procedure was applied to adjust for false discovery rate; the level of statistical significance was set at $p<0.05$.

\section{Results}

FAERS files from 2004 Q1 to 2016 Q3 contain 2589 DKA reports with an SGLT2i. Among individuals with a FAERS report listing an SGLT2i as suspect or concomitant, $12.0 \%$ had a report for DKA, compared with $0.12 \%$ among individuals with a report filed for other drugs (Table 1). This would generate a large PRR (99.6) because the background population is diluted by reports in non-diabetic individuals. Therefore, the analysis was refined by retaining only reports with an expanded diabetes indication (ESM Methods). This yielded 2028 DKA reports with SGLT2i, meaning that 561 reports $(21.7 \%)$ did not include diabetes as an indication. This is similar to the percentage of all DKA reports in the FAERS without a diabetes-related indication (22.3\%). DKA was present in $13.9 \%$ of diabetic individuals with a report for SGLT2i vs $0.54 \%$ of diabetic individuals with a report for other drugs (PRR 25.7). When only the type 1 diabetes indication was considered, the PRR was 57.3. As expected, the most common drugs listed concomitantly with SGLT2 $\mathrm{i}$ in DKA reports were metformin and insulin (ESM Table 1). Concomitant conditions were retrieved as indications for concomitant drugs: out of 13 that showed significant disproportionality, 11 were less prevalent in DKA reports with vs those without SGLT2i (ESM Table 2).

The number of annual adverse events filed to the FDA is steadily increasing and AERSMine analyses all queried adverse event reports (including pre-marketing) without selecting a specific time window. This may inflate PRR for drugs that entered the market after 2004. To overcome this bias and retrieve more details, we directly mined FAERS files between 2014 Q1 and 2016 Q3 and found 2397 DKA reports with an SGLT2i (see the list of FDA reports analysed in the study, available in the figshare repository, $10.6084 / \mathrm{m} 9$. figshare.4903211). These included 680 DKA reports among 5694 individuals with reports for dapagliflozin (11.9\%), 1362 DKA reports among 14,117 for canagliflozin (9.6\%) and 355 DKA reports among 2719 for empagliflozin (13.1\%), whereas $1.34 \%$ of reports for other drugs included DKA. Overall, the PRR of DKA in reports with vs those without an SGLT2i was 7.9. As reports with and without SGLT2i were temporally matched in this analysis, the PRR is more accurate, but it only reflects disproportionality within the FAERS and does not inform on the risk in the real world.

Temporal trends in the number of DKA reports and worldwide distribution are shown in Fig. 1. The majority of cases (76.2\%) were reported by healthcare professionals, but $19.5 \%$ 
Table 1 Frequency of DKA reports in which an SGLT2i was listed among suspect or concomitant drugs in the FAERS

\begin{tabular}{|c|c|c|c|c|c|}
\hline \multirow[t]{2}{*}{ DKA report } & \multicolumn{5}{|c|}{ Drug listed as suspect or concomitant } \\
\hline & Dapagliflozin & Canagliflozin & Empagliflozin & Any SGLT2i & $\begin{array}{l}\text { No SGLT2i } \\
\text { (any other non-SGLT2i drug) }\end{array}$ \\
\hline \multicolumn{6}{|l|}{ All FAERS } \\
\hline Unique patients & 5696 & 13,253 & 2783 & 21,636 & $8,532,895$ \\
\hline No. of DKA reports & 752 & 1466 & 378 & 2589 & 10,247 \\
\hline Rate/1000 & 131.8 & 110.6 & 135.8 & 119.4 & 1.2 \\
\hline Safety signal (IC) & 4.6 & 4.4 & 4.7 & 4.4 & Reference \\
\hline $\operatorname{PRR}(95 \% \mathrm{CI})$ & $109.9(102.6,117.8)$ & $92.3(87.6,97.2)$ & $113.7(103.3,125.1)$ & $99.6(95.6,103.8)$ & Reference \\
\hline \multicolumn{6}{|l|}{ Diabetes indication } \\
\hline Unique patients & 3876 & 8987 & 1845 & 14,639 & 427,085 \\
\hline No. of DKA reports & 548 & 1187 & 302 & 2028 & 2299 \\
\hline Rate/1000 & 141.4 & 132.1 & 163.7 & 138.5 & 5.4 \\
\hline Safety signal (IC) & 3.1 & 2.8 & 3.3 & 2.6 & Reference \\
\hline PRR $(95 \%$ CI $)$ & $26.3(24.1,28.7)$ & $24.5(22.9,26.2)$ & $30.4(27.2,34.0)$ & $25.7(24.3,27.3)$ & Reference \\
\hline \multicolumn{6}{|l|}{ Type 1 diabetes } \\
\hline Unique patients & 128 & 297 & 48 & 472 & 16,938 \\
\hline No. of DKA reports & 85 & 206 & 26 & 317 & 201 \\
\hline Rate/1000 & 664.1 & 693.6 & 541.7 & 671.6 & 11.9 \\
\hline Safety signal (IC) & 3.7 & 3.4 & 3.7 & 3.1 & Reference \\
\hline PRR $(95 \%$ CI) & $56.6(47.1,68.1)$ & $59.1(50.6,60.2)$ & $46.2(34.4,62.0)$ & $57.3(49.2,66.6)$ & Reference \\
\hline \multicolumn{6}{|l|}{ Custom search } \\
\hline Unique patients & 5694 & 14,117 & 2719 & 22,530 & 141,823 \\
\hline No. of DKA reports & 680 & 1362 & 355 & 2397 & 1930 \\
\hline Rate/1000 & 119.4 & 96.4 & 130.5 & 106.4 & 13.6 \\
\hline Safety signal (IC) & 1.9 & 1.5 & 2.1 & 1.4 & Reference \\
\hline PRR $(95 \%$ CI) & $8.9(8.2,9.7)$ & $7.2(6.7,7.7)$ & $9.7(8.7,10.8)$ & $7.9(7.5,8.4)$ & Reference \\
\hline
\end{tabular}

Different analyses are reported according to the queried indication. Analyses of all diabetes or type 1 diabetes FAERS files were performed using AERSMine. The custom search was performed to derive detailed data on all DKA cases listing an SGLT2i as suspect or concomitant from 2014 Q1 to 2016 Q3. The term 'unique patients' is used because individuals can have more than one DKA report in the FAERS. The sum of the numbers of DKA reports for each SGLT2i may exceed the number of DKA reports for any SGLT2i because reports can list more than one SGLT2i. The information component (IC) is a safety signal logarithmic measure of disproportionality and was calculated as described in [7]. The PRR is analogous to relative risk in a cohort study, i.e. the ratio of proportion of the adverse event in the 'exposed reports' to the proportion in the 'unexposed reports'

were reported by consumers. Overall, $47.7 \%$ of reports included type 2 diabetes as a drug indication, $18.5 \%$ other diabetesrelated diagnoses, and $11.4 \%$ type 1 diabetes. As for all DKA in the FAERS, $>20 \%$ of reports had no diabetes-related indication. There was no relevant change over time in the distribution of diagnoses or type of SGLT2i (Fig. 1c, d). Duration of treatment before the event was available for only $27.3 \%$ of reports and was extremely variable (median [interquartile range] 49.5 [12182] days; range 1 day to $>8$ years). Average demographic characteristics (ESM Table 3) indicate a significant predominance of women (female:male ratio $1.21 ; p=0.002$ ), and an extremely wide range of age (14-90 years) and body weight (31.4-192.3 kg). No meaningful differences were observed in demographic characteristics according to type of SGLT2i or reporting source. As expected, mean age was significantly lower when the indication was type 1 diabetes. Outcome data were available for $99 \%$ of DKA reports: most resulted in hospitalisation, or were deemed to be life threatening or serious. Thirty-seven cases (1.54\%) were fatal: 17 with dapagliflozin, 14 with canagliflozin and six with empagliflozin. There were no demographic differences between fatal and non-fatal cases. There were no consistent trends in DKA outcome over time or in relation to type of SGLT2i (Fig. 1e, f).

\section{Discussion}

FDA and European Medicines Agency warnings of SGLT2iassociated DKA originated from just a few dozen cases [3, 4]. These warnings solicited clinicians to report similar episodes, such that the number of accrued cases is now impressive. Although stimulated reporting involves a minority of drugs 


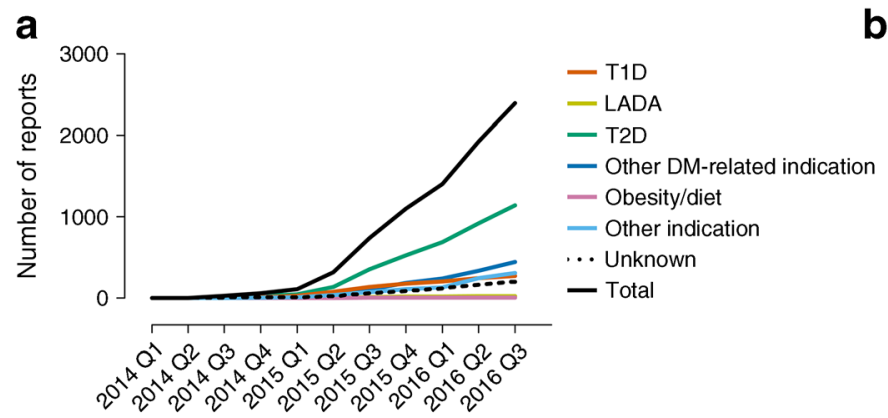

b SGLT2i and DKA in the world: 2014 Q1 to 2016 Q3

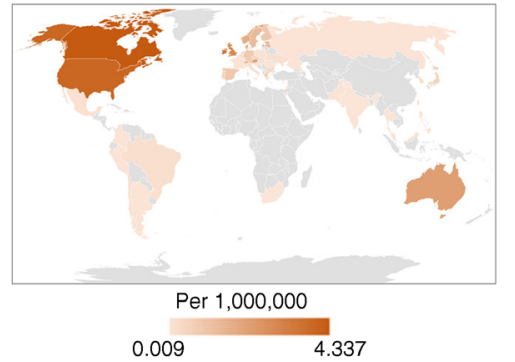

C

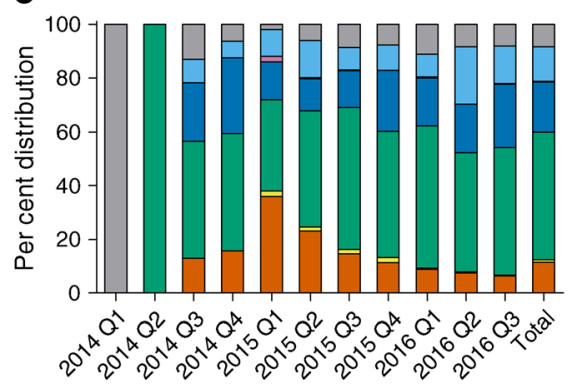

e

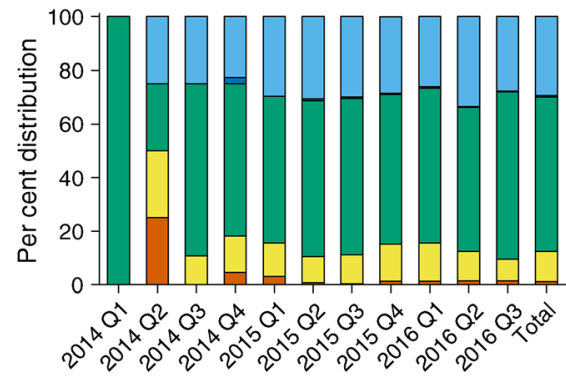

Fig. 1 Frequency and distribution of the 2397 DKA reports in which an SGLT2i was listed among suspect or concomitant drugs in the FAERS. (a) Total number of accrued reports from 2014 Q1 to 2016 Q3 and numbers divided by indication. (b) Geographic distribution of reporting country per million inhabitants. (c) Per cent distribution of indications in DKA

in the FAERS [9], SGLT2i-associated DKA may be an exception. Therefore, our data do not imply that the DKA risk in patients taking SGLT2i in the real world is increasing. In fact, FAERS data prime pharmacovigilance actions but do not inform on the true risk in clinical practice, which can only be inferred from clinical trials or cohort studies. Other caveats apply to FAERS data because most events are reported spontaneously, sometimes without validation by healthcare professionals, and there is no definite demonstration of a causal relationship between drug exposure and the adverse event. FAERS data are nonetheless useful to compare rates and describe overall demographic characteristics, associated drugs and conditions, and outcomes. The higher DKA disproportionality for SGLT2i in type 1 diabetes vs other diabetes indications may simply reflect the intrinsic susceptibility of type 1 diabetes to DKA. Although the proportion of d

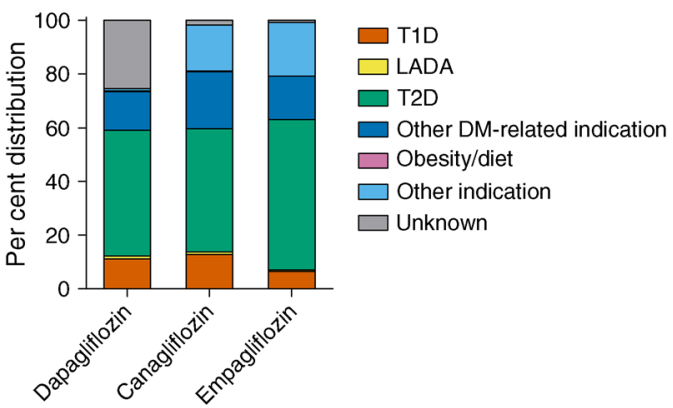

f

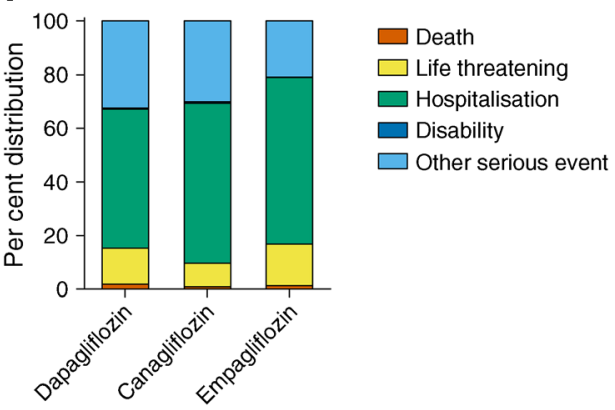

reports with an SGLT2i from 2014 Q1 to 2016 Q3. (d) Per cent distribution of indication by type of SGLT2i. (e) Distribution of outcomes of DKA reports with an SGLT2i from 2014 Q1 to 2016 Q3. (f) Distribution of outcomes by type of SGLT2i listed in the DKA reports

diabetes-unrelated indications was similar in DKA reports with and without SGLT2i, indications for SGLT2i use in DKA reports were highly heterogeneous. Furthermore, some DKA episodes were reported for very young, very old or severely underweight individuals, suggesting that SGLT2i may have been used inappropriately. The highly variable duration of SGLT2i treatment indicates that individual susceptibility is unlikely to explain DKA occurrence, making the focus on precipitating factors mandatory. The lower prevalence of several concomitant indications is consistent with the notion that SGLT2i-associated DKA may lack typical triggers, but no clear signal for novel triggers has emerged. At present, reasons for the observed sex imbalance are unclear. As infection can trigger DKA and women are more susceptible to SGLT2i-induced genitourinary tract infection, an eventual link between genitourinary tract infection and DKA should 
be scrutinised. Finally, the very low mortality rate of DKA reports involving SGLT2i compared with other DKA series $[10]$ is reassuring on this potentially life-threatening condition.

In conclusion, we provide the largest analysis of DKA reports with SGLT2i. As disproportionality may reflect stimulated reporting, these data are not expected to drive changes in the regulatory position on SGLT2i. No specific prescribing guide emerges, but appropriateness remains key to minimise the risk of SGLT2i-associated DKA. More narrative case series will help our understanding of the spectrum of this adverse event and its precipitating factors, while cohort studies are needed to ascertain the true risk in clinical practice.

Acknowledgements We wish to acknowledge the assistance of M. Sarangdhar (Cincinnati Children's Hospital Medical Center, Cincinnati, $\mathrm{OH}$, USA) for the analyses on AERSMine.

Data availability A list of FDA reports analysed in the study is available in the figshare repository, $10.6084 / \mathrm{m} 9$.figshare.4903211. Other data are available from the corresponding author on reasonable request.

Funding This research received no specific grant from any funding agency in the public, commercial or not-for-profit sectors.

Duality of interest GPF and AA have received grant support, consultancy or lecture fees from SGLT2i manufacturers. BMB declares no duality of interest associated with this manuscript.

Contribution statement GPF, BMB and AA contributed substantially to the conception and design of the study, data collection and/or analysis, and writing or revising of the manuscript. All authors approved the final version of the manuscript to be published. GPF is the guarantor of this work.

\section{References}

1. Zinman B, Wanner C, Lachin JM et al (2015) Empagliflozin, cardiovascular outcomes, and mortality in type 2 diabetes. $\mathrm{N}$ Engl $\mathrm{J}$ Med 373:2117-2128

2. Cherney DZ, Perkins BA (2014) Sodium-glucose cotransporter 2 inhibition in type 1 diabetes: simultaneous glucose lowering and renal protection? Can J Diabetes 38:356-363

3. U.S. Food and Drug Administration (2015) Drug safety communications. FDA warns that SGLT2 inhibitors for diabetes may result in a serious condition of too much acid in the blood. Available from: www.fda.gov/downloads/Drugs/DrugSafety/UCM446954.pdf. Accessed 6 Apr 2017

4. European Medicines Agency (2015) Review of diabetes medicines called SGLT2 inhibitors started: risk of diabetic ketoacidosis to be examined. Available from: www.ema.europa.eu/docs/en_GB/ document_library/Referrals_document/SGLT2_inhibitors_20/ Procedure started/WC500187926.pdf. Accessed 6 Apr 2017

5. Ferrannini E, Baldi S, Frascerra S et al (2016) Shift to fatty substrate utilization in response to sodium-glucose cotransporter 2 inhibition in subjects without diabetes and patients with type 2 diabetes. Diabetes 65:1190-1195

6. Dimitriadis GK, Manjunatha R, Harrold C (2016) Latent autoimmune diabetes in an adult initially thought to have type 2 diabetes mellitus, diagnosed after developing diabetic ketoacidosis secondary to the use of sodium glucose co-transporter 2 inhibitors. Diabetes 65:A299

7. Almenoff JS, Pattishall EN, Gibbs TG et al (2007) Novel statistical tools for monitoring the safety of marketed drugs. Clin Pharmacol Ther 82:157-166

8. Sarangdhar M, Tabar S, Schmidt C et al (2016) Data mining differential clinical outcomes associated with drug regimens using adverse event reporting data. Nat Biotechnol 34:697-700

9. Hoffman KB, Demakas AR, Dimbil M, Tatonetti NP, Erdman CB (2014) Stimulated reporting: the impact of US Food and Drug Administration-issued alerts on the Adverse Event Reporting System (FAERS). Drug Saf 37:971-980

10. Kamata Y, Takano K, Kishihara E et al (2017) Distinct clinical characteristics and therapeutic modalities for diabetic ketoacidosis in type 1 and type 2 diabetes mellitus. J Diabetes Complicat 31: $468-472$ 